\title{
Polarized Partition Relations for Ordinal Numbers
}

\author{
P. ERdös, A. Hajnal, and E. C. MilneR*
}

\section{Introduction}

There have been several instances where some particularly well-chosen symbol has enhanced the development of a branch of mathematics, and the partition symbol

$$
\alpha \rightarrow\left(\alpha_{0}, \alpha_{1}\right)^{r}
$$

invented by Richard Rado is a case in point. By definition, (1.1) means that the following relation between the ordinal (or cardinal) numbers $\alpha, \alpha_{0}, \alpha_{1}$ holds: If $A$ is an ordered set of order type $\alpha$ (we shall write tp $A=\alpha$ ) and if $[A]^{r}=\{X \subset A:|X|=r\}$ is partitioned in any way into two sets $K_{0}$ and $K_{1}$, then there are $\rho<2$ and $A^{\prime} \subset A$ such that $\operatorname{tp} A^{\prime}=\alpha_{\rho}$ and $\left[A^{\prime}\right]^{r} \subset K_{\rho}$. Erdös and Rado were the first to realize that a large number of seemingly unrelated problems in set theory could be reduced to a question of deciding whether or not some partition relation like (1.1) holds. In (5) and (6) they began a systematic study of these relations and laid the foundations of what they called $a$ partition calculus to serve as a kind of unifying principle in set theory. Since these two pioneer papers several others have been written on the subject. In particular we refer to the long paper by Erdös, Hajnal, and Rado (3) which contains an almost complete analysis for partition relations involving infinite cardinal numbers. Rado's compact symbol (1.1), which reveals at a glance the whole content of a fairly complicated combinatorial statement, proved to be particularly convenient and flexible for the development of this calculus. Apart from the merit of compactness, the symbol enjoys other advantages. The negation of any statement (1.1) is conveniently expressed by replacing the arrow $\rightarrow$ by a non-arrow $\rightarrow$. The symbol has the following obvious monotonicity properties, if $\alpha^{\prime} \geqslant \alpha, \beta^{\prime} \leqslant \beta$ and $\gamma^{\prime} \leqslant \gamma$, then (1.1) implies that

$$
\alpha^{\prime} \rightarrow\left(\beta^{\prime}, \gamma^{\prime}\right)^{r} \text {. }
$$

The arrow in (1.1) separates the two kinds of monotonicity involved and this is

* Research supported by NRC grant A-5198. Part of this paper was written at the Vancouver branch of the Canadian Mathematical Congress 1969 Summer Research Institute. 
helpful in recognizing which relations are best possible. Finally, the symbol readily lends itself to a number of interesting generalizations (see (6) and (3)).

In this paper we investigate one of these generalizations, the so-called polarized partition symbol. We consider only the simplest of such relations, namely those of the form

$$
\left\{\begin{array}{l}
\alpha \\
\beta
\end{array}\right\} \rightarrow\left\{\begin{array}{ll}
\alpha_{0} & \alpha_{1} \\
\beta_{0} & \beta_{1}
\end{array}\right\}^{1,1}
$$

By definition, this means that: If $A$ and $B$ are ordered sets, $\operatorname{tp} A=\alpha, \operatorname{tp} B=\beta$, and if the cartesian product $A \times B$ is partitioned in any way into two sets $K_{0}$ and $K_{1}$, then there are $\rho<2$ and sets $A^{\prime} \subset A, B^{\prime} \subset B$ such that $\operatorname{tp} A^{\prime}=\alpha_{\rho}$, $\operatorname{tp} B^{\prime}=\beta_{\rho}$ and $A^{\prime} \times B^{\prime} \subset K_{\rho}$. If one considersinstead, partitions of $[A]^{r} \times[B]^{s}$ for arbitrary integers $r, s$, the corresponding relation is represented by replacing the exponents 1,1 in (1.2) by $r, s$; these more general polarized relations clearly include the ordinary partition relations (1.1). Since we only consider relations with the exponents 1,1 , for the remainder of this paper we shall omit these from (1.2) and simply write

$$
\left\{\begin{array}{l}
\alpha \\
\beta
\end{array}\right\} \rightarrow\left\{\begin{array}{ll}
\alpha_{0} & \alpha_{1} \\
\beta_{0} & \beta_{1}
\end{array}\right\}
$$

Note that, as for the ordinary partition symbol, the negation of (1.2) is expressed by replacing $\rightarrow$ by $\rightarrow$. Also, we have the same monotonicity properties: if $\alpha^{\prime} \geqslant \alpha, \beta^{\prime} \geqslant \beta, \alpha_{\rho}^{\prime} \leqslant \alpha_{\rho}, \beta_{\rho}^{\prime} \leqslant \beta_{\rho}(\rho<2)$, then (1.2) implies that

$$
\left\{\begin{array}{l}
\alpha^{\prime} \\
\beta^{\prime}
\end{array}\right\} \rightarrow\left\{\begin{array}{ll}
\alpha_{0}^{\prime} & \alpha_{1}^{\prime} \\
\beta_{0}^{\prime} & \beta_{1}^{\prime}
\end{array}\right\} \text {. }
$$

Polarized partition relations were first introduced in (6), and in (3) a number of these relations involving cardinal numbers were established. As we already remarked, the theory for the ordinary partition relations involving cardinal numbers is fairly complete, but for polarized relations the situation is very different. There remain unsolved problems involving only the smallest transfinite cardinal numbers. For example, it is not known if the relation

$$
\left\{\begin{array}{l}
\aleph_{1} \\
\aleph_{2}
\end{array}\right\} \rightarrow\left\{\begin{array}{ll}
\aleph_{1} & \aleph_{1} \\
\aleph_{0} & \aleph_{0}
\end{array}\right\}
$$

is true or false. In this paper we shall establish relations of the form (1.2) which involve ordinal numbers.

As a starting point for our investigation we mention the simple, but slightly surprising, negative relation

$$
\lambda+\left(\omega_{\alpha}^{n}\right)_{n<\omega}^{1} \quad\left(\lambda<\omega_{\alpha+1}\right),
$$

proved by Milner and Rado (9). This asserts that, if $\operatorname{tp} S=\lambda<\omega_{\alpha+1}$, then $S$ 
is the union of $\aleph_{0}$ 'small' sets $A_{n}(n<\omega)$, i.e.

$$
S=\bigcup_{n<\omega} A_{n}
$$

and tp $A_{n}<\omega_{\alpha}^{n}(n<\omega)$. If we put $B_{n}=A_{0} \cup \ldots \cup A_{n}(n<\omega)$, then the sets $B_{n}$ are also 'small' ( $\operatorname{tp} B_{n}<\omega_{\alpha}^{n}$ ) and the union of any $\aleph_{0}$ of these is the whole set $S$. This fact may be expressed by means of a negative polarized relation

$$
\left\{\begin{array}{c}
\omega \\
\lambda
\end{array}\right\} \rightarrow\left\{\begin{array}{cc}
1 & \omega \\
\omega_{\alpha}^{\omega} & 1
\end{array}\right\}\left(\lambda<\omega_{\alpha+1}\right) .
$$

From (1.3), Hajnal (see (2)) deduced the following seemingly paradoxial theorem: If $\operatorname{tp} S=\lambda<\omega_{2}$, then there are $\aleph_{1}$ subsets $F_{\mu}\left(\mu<\omega_{1}\right)$ of $S$ such that tp $F_{\mu}<\omega_{1}{ }^{\omega+2}$ (i.e. the sets are 'small') and the union of any $\aleph_{0}$ of these $F_{\mu}$ is the whole set $S$. This is equivalent to the relation

$$
\left\{\begin{array}{c}
\omega_{1} \\
\lambda
\end{array}\right\} \leftrightarrow\left\{\begin{array}{ll}
1 & \omega \\
\omega_{1}{ }^{\omega+2} & 1
\end{array}\right\} \quad\left(\lambda<\omega_{2}\right) .
$$

In this paper we establish some analogous relations. We only consider relations (1.2) involving ordinal numbers of cardinal $\aleph_{1}$ and for the special case in which $\alpha=\omega_{1}, \beta=\omega_{1}{ }^{\gamma}$ and $\alpha_{0}=1$ our discussion is complete. Some of our results do generalize to ordinals of higher cardinality, but new difficulties are encountered even in the case of $\aleph_{2}$ and a discussion of these results must be left to a later paper.

In contrast to (1.4) we show (Theorem 1) that

$$
\left\{\begin{array}{l}
\omega_{1} \\
\omega_{1}{ }^{\gamma}
\end{array}\right\} \rightarrow\left\{\begin{array}{ll}
1 & \alpha \\
\xi & \beta
\end{array}\right\}
$$

where $\xi<\omega_{1}{ }^{\omega+2} \leqslant \omega_{1}{ }^{\gamma}<\omega_{2}, \alpha<\omega_{1}$ and $\beta<\omega_{1}{ }^{\gamma}$. It follows from (1.4) that the condition $\xi<\omega_{1}{ }^{\omega+2}$ is necessary in (1.5). Also, the trivial cardinal relation $(6,21.2)$

$$
\left\{\begin{array}{l}
\omega_{1} \\
\omega_{1}
\end{array}\right\} \rightarrow\left\{\begin{array}{rr}
1 & \omega_{1} \\
\omega_{1} & 1
\end{array}\right\}
$$

shows that (1.5) is false when $\alpha=\omega_{1}$. In general, the condition $\beta<\omega_{1}{ }^{\gamma}$ is also necessary for (1.5) since (Theorem 6)

$$
\left\{\begin{array}{l}
\omega_{1} \\
\omega_{1}^{\gamma}
\end{array}\right\} \rightarrow\left\{\begin{array}{cc}
1 & \omega \\
\omega_{1}^{\omega+1}+1 & \omega_{1}^{\gamma}
\end{array}\right\}
$$

if $\dagger \operatorname{co}\left(\omega_{1}^{\gamma}\right)=\omega_{1}$ and $\gamma<\omega_{2}$. On the other hand, if $c o\left(\omega_{1}^{\gamma}\right)=\omega$, then it is $\dagger$ We write $\operatorname{co}(\lambda)$ to denote the least ordinal number which is cofinal with $\lambda$. Thus $\operatorname{co}(\lambda)$ is either 1 or an initial ordinal. 
possible to strengthen (1.5), i.e. (Theorem 2)

$$
\left\{\begin{array}{c}
\omega_{1} \\
\omega_{1}^{\gamma}
\end{array}\right\} \rightarrow\left\{\begin{array}{cc}
1 & \alpha \\
\xi & \omega_{1}^{\gamma}
\end{array}\right\}
$$

if $\xi<\omega_{1}{ }^{\omega+2}<\omega_{1}{ }^{\gamma}, \alpha<\omega_{1}$ and $\operatorname{co} \gamma=\omega$. Note that (1.7) is best possible since (Theorem 3 )

$$
\left\{\begin{array}{c}
\omega_{1} \\
\omega_{1}^{\gamma}
\end{array}\right\} \rightarrow\left\{\begin{array}{cc}
1 & \alpha \\
\omega_{1}^{\omega+1} & \omega_{1}^{\gamma}
\end{array}\right\}
$$

holds if $\omega+1 \leqslant \gamma<\omega_{2}$.

We shall prove (Theorem 4 ) that

$$
\left\{\begin{array}{c}
\omega_{1} \\
\omega_{1}{ }^{\gamma}
\end{array}\right\} \rightarrow\left\{\begin{array}{cc}
k & \alpha \\
\omega_{1}{ }^{\gamma} & \omega_{1}{ }^{\gamma}
\end{array}\right\}
$$

holds for $k<\omega, \alpha<\omega_{1}$ and $\gamma \leqslant \omega+1$. This is stronger than (1.9) when $\gamma=\omega+1$. It is not possible to replace $k$ by $\omega$ in (1.10) since it is known (3, Theorem 32) that

$$
\left\{\begin{array}{c}
\omega_{1} \\
\omega_{1}
\end{array}\right\} \rightarrow\left\{\begin{array}{cc}
\omega & \omega \\
\omega_{1} & \omega_{1}
\end{array}\right\}
$$

However, this raises the question whether one can replace 1 by any integer $k$ in (1.5), (1.8) and (1.9). This is not possible in the case of (1.9) since we can prove (see (1.16)) with the continuum hypothesis $\dagger$ that

$$
\left\{\begin{array}{c}
\omega_{1} \\
\omega_{1}{ }^{\gamma}
\end{array}\right\} \rightarrow\left\{\begin{array}{cc}
2 & \omega \\
\omega_{1}{ }^{\omega} & \omega_{1}{ }^{\gamma}
\end{array}\right\}
$$

if $\omega+2 \leqslant \gamma<\omega_{2}$ and $\operatorname{co}\left(\omega_{1}{ }^{\gamma}\right)=\omega_{1}$. We do not know the status of (1.5) and (1.8) in this connection.

Problem 1. Is the relation

$$
\left\{\begin{array}{c}
\omega_{1} \\
\omega_{1}^{\omega 2}
\end{array}\right\} \rightarrow\left\{\begin{array}{cc}
2 & \omega \\
\omega_{1}^{\omega} & \omega_{1}{ }^{\omega 2}
\end{array}\right\}
$$

true or false?

There is another problem of this kind (see $\$ 4$ ) which we cannot settle.

$\dagger$ Where we use the continuum hypothesis to prove a result, we prefix the statement by $\left(^{*}\right)$ for easier recognition. 
Problem 2. Does the relation

$$
\left\{\begin{array}{c}
\omega_{1} \\
\omega_{1}{ }^{\omega+2}
\end{array}\right\} \rightarrow\left\{\begin{array}{cc}
3 & \alpha \\
\omega_{1}{ }^{\omega+1} & \xi
\end{array}\right\}
$$

hold for $\alpha<\omega_{1}$ and $\xi<\omega_{1}{ }^{\omega+2}$ ?

It follows as a special case of a result proved in (2, Theorem 1) that

$$
\left\{\begin{array}{c}
\omega_{1} \\
\omega_{1}{ }^{\gamma}
\end{array}\right\} \rightarrow\left\{\begin{array}{ll}
1 & \omega_{1} \\
\alpha & \omega_{1}{ }^{\gamma}
\end{array}\right\}
$$

if $\alpha<\omega_{1}, \gamma<\omega_{2}$ and $\operatorname{co}\left(\omega_{1}{ }^{\gamma}\right)=\omega_{1}$. We also showed (2, Theorem 2) that

$$
\left\{\begin{array}{c}
\omega_{1} \\
\omega_{1}^{\gamma}
\end{array}\right\} \rightarrow\left\{\begin{array}{cc}
1 & \omega_{1} \\
\omega+1 & \omega_{1}^{\gamma}
\end{array}\right\}
$$

if $c o\left(\omega_{1}{ }^{\gamma}\right)=\omega$. The method we used to prove (1.13) is very different from the methods used in this paper. We shall not give the details, but with the same method used in (2) one can also show that

$$
\left\{\begin{array}{c}
\omega_{1} \\
\omega_{1}^{\gamma}
\end{array}\right\} \rightarrow\left\{\begin{array}{cc}
1 & \omega_{1} \\
\omega & \omega_{1}^{\gamma}
\end{array}\right\} \quad\left(\gamma<\omega_{2}\right) .
$$

We mention these results because these three relations (1.13)-(1.15), together with (1.4), (1.5), (1.7), (1.8), (1.9) and (1.10) give a complete analysis of the symbol (1.2) for the case $\alpha=\omega_{1}, \beta=\omega_{1}^{\gamma}$ and $\alpha_{0}=1$.

In $\S 6$ we establish some strong negative results. Using the continuum hypothesis we prove (Theorem 7) that, for $\gamma<\omega_{2}$ and $c o\left(\omega_{1}{ }^{\gamma}\right)=\omega_{1}$,

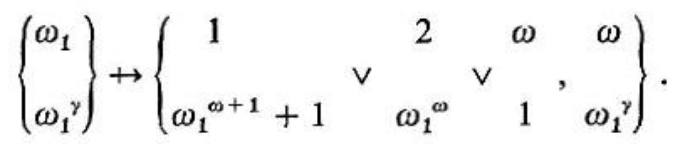

Here we are using the partition symbol with alternatives (for the definition see §2). An equivalent formulation of (1.16) is the following. If $\operatorname{tp} S=\omega_{1}{ }^{\gamma}<\omega_{2}$ and the continuum hypothesis is assumed, then there is a family of $\aleph_{1}$ sets $F_{\mu} \subset S\left(\mu<\omega_{1}\right)$ such that (i) $\operatorname{tp} F_{\mu} \leqslant \omega_{1}{ }^{\omega+1}$ (i.e. 'small' sets), (ii) $\operatorname{tp} F_{\mu} \cap F_{\lambda}<\omega_{1}{ }^{\omega}$ if $\lambda \neq \mu$ (i.e. the intersection of any pair is 'very small'), (iii) each point of $S$ belongs to only finitely many of the sets $F_{\mu}$ and (iv) the union of any $\aleph_{0}$ of these sets is nearly all of $S$, i.e.

$$
\operatorname{tp}\left(S-\bigcup_{\mu \in N} F_{\mu}\right)<\omega_{1}^{\gamma}
$$

for any infinite set of indices $N$. Using a different kind of notation, we proved (1, Theorem 10.14) that

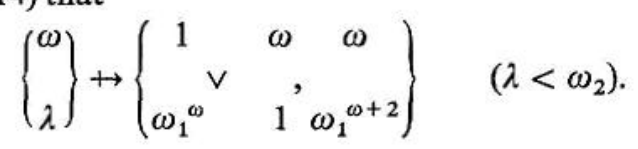


In Theorem 8 we establish the analogous result

$$
\left\{\begin{array}{c}
\omega_{1} \\
\lambda
\end{array}\right\} \leftrightarrow\left\{\begin{array}{cccc}
1 & \omega & \omega \\
\omega_{1}{ }^{\omega+2} & \vee & & \\
& 1 & \omega_{1}{ }^{\omega+2}
\end{array}\right\} \quad\left(\lambda<\omega_{2}\right) .
$$

This last relation is also best possible since (see §7)

$$
\left\{\begin{array}{c}
\omega_{1} \\
\omega_{1}^{\gamma}
\end{array}\right\} \rightarrow\left\{\begin{array}{cccc}
1 & & \omega & \omega \\
& \vee & & , \\
\omega_{1}^{\gamma} & & 1 & \xi
\end{array}\right\}
$$

holds if $\xi<\omega_{1}{ }^{\omega+2}<\omega_{1}^{\gamma}<\omega_{2}$.

\section{Notation and preliminary results}

Unless stated otherwise small Latin and Greek letters denote ordinal numbers. Capital letters denote sets and, in particular,

$$
W_{1}=\left\{v: v<\omega_{1}\right\}
$$

is the set of countable ordinals. The obliterator sign $\wedge$ above any symbol means that that symbol is to be disregarded, e.g. $\left\{x_{0}, \ldots, \hat{x}_{\lambda}\right\}=\left\{x_{v}: v<\lambda\right\}$. We write $\left\{x_{0}, \ldots, \hat{x}_{\lambda}\right\}<$ to indicate that the set $\left\{x_{0}, \ldots, \hat{x}_{\lambda}\right\}$ is ordered so that $x_{\mu}<x_{v}$ for $\mu<v<\lambda$. Similarly, $\left\{x_{0}, \ldots, \hat{x}_{\lambda}\right\} \neq$ means that $x_{\mu} \neq x_{v}$ for $\mu<v<\lambda$. The order type of an ordered set $S$ is denoted by tp $S$. If $X, Y \subset S$, then $X<Y$ means that $x<y$ holds for all $x \in X$ and $y \in Y$. If $S$ is the disjoint union of sets $S_{v}(v<\lambda)$ and $S_{\mu}<S_{v}$ holds for $\mu<v<\lambda$, then we write

$$
S=S_{0} \cup \ldots \cup \hat{S}_{\lambda}(<) \text { or } S=\bigcup_{v<\lambda} S_{v}(<) .
$$

A subset $X$ of $S$ is cofinal with $S$ if $X<\{\alpha\}$ is false for every $\alpha \in S$. We define $\operatorname{co}(\lambda)$ to be the least ordinal $\mu$ such that tp $T=\mu$ for some cofinal subset $T$ of $\{v: v<\lambda\}$.

The cardinal of $S$ is $|S|$, and $[S]^{r}=\{X \subset S:|X|=r\}$ for any cardinal number $r$. The partition symbol

$$
\alpha \rightarrow\left(\alpha_{v}\right)^{r<\lambda}
$$

means: if tp $S=\alpha$ and $[S]^{r}=\bigcup_{v<\lambda} K_{v}$, then there are $\mu<\lambda$ and $A \subset S$ such that tp $A=\alpha_{\mu}$ and $[A]^{r} \subset K_{\mu}$. If $\alpha_{v}=\beta$ for all $v<\lambda$ we write (2.1) as

$$
\alpha \rightarrow(\beta)_{\lambda} \text {. }
$$


We need the following simple relations of the form (2.1) with $r=1$ (see (9)):

$$
\begin{array}{ll}
\omega_{1}{ }^{n} \rightarrow\left(\omega_{1}{ }^{n}\right)_{\lambda}{ }^{1} & \text { if } \lambda<\omega_{1} \text { and } n<\omega, \\
\omega_{1}{ }^{\rho} \rightarrow\left(\omega_{1}{ }^{1}\right)_{1} & \text { if } l<\omega, \\
\xi \leftrightarrow\left(\omega_{1}{ }^{1}\right)_{n<\omega} & \text { if } \xi<\omega_{2} .
\end{array}
$$

These results generalize to ordinal numbers of arbitrary cardinality, but we do not use this fact.

A set mapping is a function $f: S \rightarrow\{X: X \subset S\}$ such that $x \notin f(x)(x \in S)$. A free set in this mapping is a subset $S^{\prime}$ of $S$ such that $x \notin f(y)$ for all $x, y \in S^{\prime}$. It was shown by Erdös and Specker (7) that, if $S=\left\{v: v<\omega_{\lambda}\right\}$ and $f$ is a set mapping on $S$ such that tp $(f(x))<\alpha\left(<\omega_{\lambda}\right)$, then there is a free set $S^{\prime} \subset S$ of type $\omega_{\lambda}$. Their proof required the generalized continuum hypothesis but Hajnal (8) showed how to eliminate this hypothesis. In (2) we pointed out a general connection between the theory of set mappings and the polarized partition relations. We need the above theorem only for the case $\lambda=1$ and this may be expressed by the relation

$$
\left\{\begin{array}{c}
\omega_{1} \\
\omega_{1}
\end{array}\right\} \rightarrow\left\{\begin{array}{ll}
1 & \omega_{1} \\
\alpha & \omega_{1}
\end{array}\right\} \quad\left(\alpha<\omega_{1}\right) .
$$

The polarized partition symbol (1.2) has already been defined. In $\S \S 6,7$ we use a slight extension of this by allowing alternative entries. Formally,

$$
\left\{\begin{array}{l}
\alpha \\
\beta
\end{array}\right\} \rightarrow\left\{\begin{array}{lllll}
\alpha_{v 1} & \alpha_{v 2} & & & \alpha_{v k_{v}} \\
\beta_{v 1} & \beta_{v 2} & & & \beta_{v k_{v}}
\end{array}\right\}_{v<\lambda}
$$

means: if $\operatorname{tp} A=\alpha, \operatorname{tp} B=\beta$ and

$$
A \times B=\bigcup_{v<\lambda} K_{v}
$$

is any partition, then there are $\mu<\lambda$ and $l<k_{\mu}$ and sets $A^{\prime} \subset A, B^{\prime} \subset B$ such that $\operatorname{tp} A^{\prime}=\alpha_{\mu l}, \operatorname{tp} B^{\prime}=\beta_{\mu l}$ and $A^{\prime} \times B^{\prime} \subset K_{\mu}$.

If $K_{0} \cup K_{1}$ is a partition of $A \times B$ then we define

$$
\begin{array}{ll}
K_{\rho}(a)=\left\{b \in B:\{a, b\} \in K_{\rho}\right\} & (a \in A), \\
K_{\rho}(b)=\left\{a \in A:\{a, b\} \in K_{\rho}\right\} & (b \in B) .
\end{array}
$$

Also, if $X \subset A \cup B$, then we write

$$
K_{\rho}(X)=\bigcup_{x \in X} K_{\rho}(x) \quad(\rho=0 \text { or } 1) .
$$

A graph is an ordered pair $G=(S, E)$ with $E \subset[S]^{2}$. The elements of $E$ are the edges of $G$. $G=(S, E)$ is a complete graph if $E=[S]^{2}$. A circuit of $G$ of 
length $k(>2)$ is a sequence $x_{1}, x_{2}, \ldots, x_{k}$ of $k$ distinct elements of $S$ such that $\left\{x_{i}, x_{i+1}\right\} \in E(1 \leqslant i \leqslant k)$, where $x_{k+1}=x_{1}$. A graph without circuits is called a forest.

\section{Some lemmas}

We establish here some simple lemmas which will be used in the next section.

LEMMA 1. Let $\alpha<\omega_{1}$ and let $M_{n} \in\left[W_{1}\right]^{\aleph_{1}}(n<\omega)$. Then there are $\mu_{n} \in M_{n}$ $(n<\omega)$ such that $\operatorname{tp}\left\{\mu_{n}: n<\omega\right\} \geqslant \alpha$.

Proof. It is enough to prove this in the case $\alpha=\omega^{\beta}$. For $\beta=0$, the result is obvious. Now assume that $\beta>0$ and use induction. We may write $\omega^{\beta}=\alpha_{0}+$ $\alpha_{1}+\ldots+\hat{\alpha}_{\omega}$, where $\alpha_{n}=\omega^{\beta_{n}}<\omega^{\beta}(n<\omega)$. Let $\{n: n<\omega\}$ be partitioned into $\aleph_{0}$ disjoint infinite sets $N_{i}(i<\omega)$. Let $l<\omega$ and suppose we have already chosen $\mu_{n} \in M_{n}$ for $n \in N_{0} \cup \ldots \cup \widehat{N}_{l}$. For $k \in N_{l}$, let

$$
M_{k}^{\prime}=\left\{\mu \in M_{k}: \mu>\mu_{n} \text { for all } n \in N_{0} \cup \ldots \cup \widehat{N}_{l}\right\} .
$$

Then $M_{k}^{\prime}$ is a cofinal subset of $W_{1}\left(k \in N_{l}\right)$. Since $N_{l}$ is infinite, it follows from the induction hypothesis that there are elements $\mu_{n} \in M_{n}^{\prime}\left(n \in N_{l}\right)$ such that

$$
\operatorname{tp}\left\{\mu_{n}: n \in N_{l}\right\} \geqslant \alpha_{l} .
$$

This defines $\mu_{n} \in M_{n}$ for all $n<\omega$. By the construction,

$$
\operatorname{tp}\left\{\mu_{n}: n<\omega\right\}=\sum_{l<\omega} \operatorname{tp}\left\{\mu_{n}: n \in N_{l}\right\} \geqslant \sum_{l<\omega} \alpha_{l}=\omega^{\beta} .
$$

We frequently use the following result.

LEMMA 2. If $T \subset S_{0} \cup S_{1} \cup \ldots \cup \hat{S}_{\omega_{1}}(<)$ and $\operatorname{tp} T<\omega_{1}{ }^{\omega+1}$, then there are $v<\omega_{1}$ and $n<\omega$ such that $\operatorname{tp}\left(T \cap S_{\rho}\right)<\omega_{1}{ }^{n}\left(v<\rho<\omega_{1}\right)$.

Proof. Suppose the lemma is false. For $\rho<\omega_{1}$ we may write $\rho=\omega \xi+n$, where $\xi=\xi(\rho)<\omega_{1}$ and $n=n(\rho)<\omega$. Suppose $\rho<\omega_{1}$ and that $v_{\sigma}<\omega_{1}$ has been defined for $\sigma<\rho$. By our assumption, there is $v_{\rho}<\omega_{1}$ such that $v_{\sigma}<v_{\rho}(\sigma<\rho)$ and tp $\left(T \cap S_{v_{\rho}}\right) \geqslant \omega_{1}^{n(\rho)}$. This defines $v_{\rho}<\omega_{1}$ for all $\rho<\omega_{1}$. From the definition we have

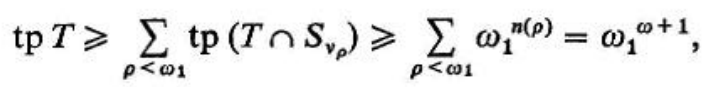

a contradiction. 
LEMMA 3. Let $S_{n}(n<\omega)$ be $\aleph_{0}$ disjoint sets and suppose that $\operatorname{tp} S_{n}=\omega_{1}^{\gamma_{n}}$, where $\omega<\gamma_{n}<\omega_{1}$ and $\operatorname{co}\left(\omega_{1}^{\gamma_{n}}\right)=\omega_{1}$. Let $\alpha<\omega_{1}$ and let $F_{\mu}\left(\mu<\omega_{1}\right)$ be $\aleph_{1}$ sets such that

$$
\operatorname{tp}\left(S_{n} \cap F_{\mu}\right)<\omega_{1}^{\omega+1} \quad\left(n<\omega ; \mu<\omega_{1}\right) .
$$

Then there is $A \subset W_{1}$ such that $\operatorname{tp} A \geqslant \alpha$ and

$$
\operatorname{tp}\left(S_{n}-\bigcup_{\mu \in A} F_{\mu}\right)=\operatorname{tp} S_{n} \quad(n<\omega) .
$$

Proof. By the hypothesis, there are $\gamma_{n v}\left(n<\omega ; v<\omega_{1}\right)$ such that

and

$$
\omega \leqslant \gamma_{n 0} \leqslant \gamma_{n 1} \leqslant \ldots \leqslant \hat{\gamma}_{n \omega_{1}}<\gamma_{n}
$$

$$
\sum_{v<\omega_{1}} \omega_{1}^{\gamma_{n v}}=\omega_{1}^{\gamma_{n}}
$$

We may write

$$
S_{n}=\bigcup_{\nu<\omega_{1}} S_{n v}(<),
$$

where tp $S_{n v}=\omega_{1}{ }^{\gamma_{n \nu}}$. By (3.1) and Lemma 2, there are $v(n, \mu)<\omega_{1}$ and $i(n, \mu)$ $<\omega\left(n<\omega ; \mu<\omega_{1}\right)$ such that

$$
\operatorname{tp}\left(S_{n v} \cap F_{\mu}\right)<\omega_{1}^{i(n, \mu)} \quad\left(v(n, \mu)<v<\omega_{1}\right) .
$$

There are sets $M_{n} \in\left[W_{1}\right]^{\mathbb{N}_{1}}(n<\omega)$ such that $M_{0} \supset M_{1} \supset \ldots$ and

$$
i(n, \mu)=i(n) \quad\left(\mu \in M_{n}\right) .
$$

By Lemma 1, there are $\mu_{n} \in M_{n}(n<\omega)$ such that $A=\left\{\mu_{n}: n<\omega\right\}$ has order type $\geqslant \alpha$. Choose $\lambda<\omega_{1}$ such that $v\left(n, \mu_{m}\right)<\lambda(m, n<\omega)$ and let

Then

$$
k(n)=\max \left\{i(n), i\left(n, \mu_{0}\right), \ldots, i\left(n, \mu_{n}\right)\right\} \quad(n<\omega) .
$$

$$
\operatorname{tp}\left(F_{\mu_{m}} \cap S_{n v}\right)<\omega_{1}^{k(n)} \quad\left(m, n<\omega ; \lambda<v<\omega_{1}\right) .
$$

Since $k(n)<\omega$, it follows from (2.2) and (2.3) that

$$
\operatorname{tp}\left(S_{n v}-\bigcup_{\mu \in A} F_{\mu}\right)=\omega_{1}^{\gamma_{n v}} \quad\left(n<\omega ; \lambda<v<\omega_{1}\right)
$$

and (3.2) follows.

LeMMA 4. Let $\omega+1 \leqslant \gamma_{0} \leqslant \gamma_{1} \leqslant \ldots \leqslant \hat{\gamma}_{\omega_{1}}<\omega_{2}$ and let

$$
\beta<\sum_{\nu<\omega_{1}} \omega_{1}^{\gamma_{\nu}}=\omega_{1}^{\gamma} \text {. }
$$


Then there are a countable set $N \subset W_{1}$ and ordinals $\beta_{v}(v \in N)$ such that

and

$$
\omega+1 \leqslant \beta_{v} \leqslant \gamma_{v}, \quad \operatorname{co}\left(\omega_{1}{ }^{\beta_{v}}\right)=\omega_{1}
$$

$$
\beta<\sum_{v \in N} \omega_{1}^{\beta_{v}} .
$$

Proof. By the hypothesis, there is $\theta$ such that $\omega+1 \leqslant \theta<\gamma$ and $\beta<\omega_{1}{ }^{\theta+1}$. If $\theta<\gamma_{v}$ for some $v<\omega_{1}$, then (3.3) holds with $N=\{v\}$ and $\beta_{v}=\theta+1$. We may therefore assume that there is $v_{0}<\omega_{1}$ such that $\gamma_{v}=\theta\left(v_{0} \leqslant v<\omega_{1}\right)$. Therefore, $\gamma=\theta+1$ and there is $\xi<\omega_{1}$ such that $\beta<\omega_{1}^{\theta} \xi$. If $\operatorname{co}\left(\omega_{1}^{\theta}\right)=\omega_{1}$, then (3.3) holds with $N=\left\{v: v_{0} \leqslant v<v_{0}+\xi\right\}$ and $\beta_{v}=\theta$. Suppose, on the other hand, that $\operatorname{co}\left(\omega_{1}{ }^{\theta}\right)=\omega$. Then there are $\theta_{n}(n<\omega)$ such that $\omega+1 \leqslant$ $\theta_{0}<\theta_{1}<\ldots<\hat{\theta}_{\omega}<\theta=\lim _{n<\omega} \theta_{n}$. In this case, (3.3) holds with

$$
N=\left\{v: v_{0} \leqslant v<v_{0}+\omega \xi\right\} \text { and } \beta_{v_{0}+\omega \rho+n}=\omega_{1}^{\theta_{n}+1} \quad(\rho<\xi ; n<\omega) \text {. }
$$

\section{Positive results}

In contrast to the negative relation

$$
\left\{\begin{array}{c}
\omega_{1} \\
\lambda
\end{array}\right\} \rightarrow\left\{\begin{array}{ll}
1 & \omega \\
\omega_{1}{ }^{\omega+2} & 1
\end{array}\right\} \quad\left(\lambda<\omega_{2}\right)
$$

proved in (2), we shall establish the following theorem.

THEOREM 1. If $\alpha<\omega_{1}, \xi<\omega_{1}^{\omega+2}, \beta<\omega_{1}^{\gamma}$ and $\omega+2 \leqslant \gamma<\omega_{1}$, then

$$
\left\{\begin{array}{l}
\omega_{1} \\
\omega_{1}^{\gamma}
\end{array}\right\} \rightarrow\left\{\begin{array}{ll}
1 & \alpha \\
\xi & \beta
\end{array}\right\} \text {. }
$$

Proof. We first prove the result for the case $\operatorname{co}\left(\omega_{1}{ }^{\gamma}\right)=\omega_{1}$.

Let $\operatorname{tp} C=\omega_{1}{ }^{\gamma}$ and let $W_{1} \times C=K_{0} \cup K_{1}$ be a partition such that $\operatorname{tp}\left(K_{0}(\mu)\right)<\xi$ for all $\mu \in W_{1}$. We have to show that there are sets $A \subset W_{1}$ and $B \subset C$ such that tp $A \geqslant \alpha, \operatorname{tp} B \geqslant \beta$ and $A \times B \subset K_{1}$.

Since $c o\left(\omega_{1}{ }^{7}\right)=\omega_{1}$, we may write

$$
C=\bigcup_{v<\omega_{1}} C_{v}(<),
$$

where tp $C_{v}=\omega_{1}^{\gamma_{\nu}}\left(v<\omega_{1}\right)$ and

$$
\omega+1 \leqslant \gamma_{0} \leqslant \gamma_{1} \leqslant \ldots \leqslant \hat{\gamma}_{\omega_{1}}<\gamma .
$$


There is $\eta<\omega_{1}$ such that $\xi<\omega_{1}{ }^{\omega+1} \eta$ and the sets

$$
F(\mu)=\left\{v<\omega_{1}: \operatorname{tp}\left(K_{0}(\mu) \cap C_{v}\right) \geqslant \omega_{1}{ }^{\omega+1}\right\} \quad\left(\mu<\omega_{1}\right)
$$

have order type less than $\eta$. Therefore, by (2.5), there are $M, N \in\left[W_{1}\right]^{N_{1}}$ such that

$$
\operatorname{tp}\left(K_{0}(\mu) \cap C_{v}\right)<\omega_{1}{ }^{\omega+1} \quad(\mu \in M ; v \in N) .
$$

By Lemma 4 there are a countable set $N_{0} \subset N$ and ordinals $\beta_{v}\left(v \in N_{0}\right)$ such that $\omega+1 \leqslant \beta_{v} \leqslant \gamma_{v}$ and $\operatorname{co}\left(\omega_{1}^{\beta_{v}}\right)=\omega_{1}\left(v \in N_{0}\right)$ and such that

$$
\beta<\sum_{v \in N_{0}} \omega_{1}^{\beta_{\nu}} .
$$

Let $S_{v}$ be a subset of $C_{v}$ of type $\omega_{1}^{\beta_{v}}\left(v \in N_{0}\right)$. Then, by (4.3) and Lemma 3, there is a set $A \subset M$ such that tp $A \geqslant \alpha$ and

$$
\operatorname{tp}\left(S_{v}-\bigcup_{\mu \in A} K_{0}(\mu)\right)=\operatorname{tp} S_{v} \quad\left(v \in N_{0}\right) .
$$

This implies that the set

$$
B=\bigcup_{v \in N_{0}} S_{v}-\bigcup_{\mu \in A} K_{0}(\mu)
$$

has type $\geqslant \beta$ and $A \times B \subset K_{1}$. This proves (4.2) for the case $c o\left(\omega_{1}^{\gamma}\right)=\omega_{1}$.

The case $c o\left(\omega_{1}{ }^{\gamma}\right)=\omega$ follows immediately from this. For, if $\operatorname{co} \gamma=\omega$ and $\beta<\omega_{1}^{\gamma}$, then $\beta<\omega_{1}{ }^{\delta+1}$ for some $\delta+1<\gamma$ and (4.2) is implied by the relation

$$
\left\{\begin{array}{c}
\omega_{1} \\
\omega_{1}^{\delta+1}
\end{array}\right\} \rightarrow\left\{\begin{array}{ll}
1 & \alpha \\
\xi & \beta
\end{array}\right\} .
$$

The condition $\xi<\omega_{1}{ }^{\omega+2}$ in Theorem 1 is necessary because of (4.1). Also, in view of the trivial relation (1.6), the condition $\alpha<\omega_{1}$ is necessary. The relation (4.2) is best possible in a third sense since (Theorem 6)

$$
\left\{\begin{array}{l}
\omega_{1} \\
\omega_{1}^{\gamma}
\end{array}\right\} \rightarrow\left\{\begin{array}{cc}
1 & \omega \\
\omega_{1}{ }^{\omega+1}+1 & \omega_{1}^{\gamma}
\end{array}\right\} \quad \text { if } \operatorname{co}\left(\omega_{1}^{\gamma}\right)=\omega_{1} .
$$

This shows that we cannot replace $\beta$ by $\omega_{1}{ }^{\gamma}$ in (4.2) when $\operatorname{co}\left(\omega_{1}{ }^{\gamma}\right)=\omega_{1}$. In the next theorem we show that (4.2) can be strengthened if $c o\left(\omega_{1}{ }^{\gamma}\right)=\omega$.

THEOREM 2. If $\alpha<\omega_{1}, \xi<\omega_{1}{ }^{\omega+2}<\omega_{1}^{\gamma}<\omega_{2}$ and $\operatorname{co}(\gamma)=\omega$, then

$$
\left\{\begin{array}{c}
\omega_{1} \\
\omega_{1}{ }^{\gamma}
\end{array}\right\} \rightarrow\left\{\begin{array}{cc}
1 & \alpha \\
\xi & \omega_{1}^{\gamma}
\end{array}\right\} .
$$

Proof. We shall prove the result by induction on $\alpha$. For $\alpha=0$ or 1 the result is obvious. Now assume that $1<\alpha^{\prime}<\omega_{1}$ and that (4.5) holds for all $\alpha<\alpha^{\prime}$. 
Since $1<\alpha^{\prime}<\omega_{1}$, there are ordinals $\alpha_{n}<\alpha^{\prime}(n<\omega)$ such that

$$
\alpha^{\prime}=\alpha_{0}+\alpha_{1}+\ldots .
$$

Also, by the hypothesis of the theorem, there are $\gamma_{n}(n<\omega)$ such that

$$
\omega<\gamma_{0}<\gamma_{1}<\ldots<\gamma=\lim _{n<\omega} \gamma_{n} .
$$

Let tp $C_{0}=\omega_{1}{ }^{\gamma}, M_{0}=W_{1}$, and let $K_{0} \cup K_{1}$ be any partition of $M_{0} \times C_{0}$ such that $\operatorname{tp}\left(K_{0}(\mu)\right)<\xi\left(\mu \in M_{0}\right)$. We will show that there are sets $A \subset M_{0}$ and $B \subset C_{0}$ such that tp $A \geqslant \alpha^{\prime}, \operatorname{tp} B=\omega_{1}{ }^{\gamma}$ and $A \times B \subset K_{1}$.

By the induction hypothesis, (4.5) holds for $\alpha=\alpha_{0}$ and hence there are sets $A_{0} \subset M_{0}$ and $D_{0} \subset C_{0}$ such that tp $A_{0}=\alpha_{0}$, tp $D_{0}=\omega_{1}{ }^{\gamma}$ and $A_{0} \times D_{0} \subset K_{1}$. Let

$$
D_{0}=E_{0} \cup C_{1}(<),
$$

where $E_{0}$ is the initial section of $D_{0}$ of type $\omega_{1}{ }^{{ }^{0}+2}$. We may write

$$
E_{0}=\bigcup_{\rho<\omega_{1}} E_{0 \rho}(<), \quad E_{0 \rho}=\bigcup_{\sigma<\omega_{1}} E_{0 \rho \sigma}(<),
$$

where tp $E_{0 \rho \sigma}=\omega_{1}{ }^{\gamma_{0}}\left(\rho, \sigma<\omega_{1}\right)$. There is $\eta<\omega_{1}$ such that $\xi<\omega_{1}{ }^{\omega+1} \eta$. Therefore, since tp $K_{0}(\mu)<\xi\left(\mu \in M_{0}\right)$ and

$$
\left\{\begin{array}{l}
\omega_{1} \\
\omega_{1}
\end{array}\right\} \rightarrow\left\{\begin{array}{cc}
1 & \omega_{1} \\
\eta & 1
\end{array}\right\}
$$

by (2.5), it follows that there are $M_{0}{ }^{\prime} \in\left[M_{0}\right]^{\aleph_{1}}$ and $\rho_{0}<\omega_{1}$ such that

$$
\operatorname{tp}\left(K_{0}(\mu) \cap E_{0 \rho_{0}}\right)<\omega_{1}{ }^{\omega+1} \quad\left(\mu \in M_{0}{ }^{\prime}\right) .
$$

Therefore, by Lemma 2, for each $\mu \in M_{0}{ }^{\prime}$ there are $p_{0}(\mu)<\omega$ and $\sigma_{0}(\mu)<\omega_{1}$ such that

$$
\operatorname{tp}\left(K_{0}(\mu) \cap E_{0 \rho_{0} \sigma}\right)<\omega_{1}{ }^{p_{0}(\mu)} \text { for } \sigma_{0}(\mu)<\sigma<\omega_{1} .
$$

There is a set $M_{1} \in\left[M_{0}^{\prime}\right]^{\mathbb{K}_{1}}$ such that $A_{0}<M_{1}$ and such that

$$
p_{0}(\mu)=p_{0}\left(\mu \in M_{1}\right) \text {. }
$$

More generally, suppose that $n<\omega$ and $C_{n} \subset C_{0}, M_{n} \subset M_{0}$ have been defined so that $\operatorname{tp} C_{n}=\omega_{1}^{\gamma}$ and $\operatorname{tp} M_{n}=\omega_{1}$. By the induction hypothesis, (4.5) holds with $\alpha=\alpha_{n}$ and so there are sets $A_{n} \subset M_{n}$ and $D_{n} \subset C_{n}$ such that $\operatorname{tp} A_{n}=\alpha_{n}$, tp $D_{n}=\omega_{1}^{\gamma}$ and $A_{n} \times D_{n} \subset K_{1}$. Let $E_{n}$ be the initial section of type $\omega_{1}^{\gamma_{n}+2}$ and let

We may write

$$
D_{n}=E_{n} \cup C_{n+1}(<) \text {. }
$$

$$
E_{n}=\bigcup_{\rho<\omega_{1}} E_{n \rho}(<) \text { and } E_{n \rho}=\bigcup_{\sigma<\omega 1} E_{n \rho \sigma}(<),
$$


where $\operatorname{tp}\left(E_{n \rho \sigma}\right)=\omega_{1}{ }^{\gamma_{n}}$. It follows from (4.6) that there are $p_{n}<\omega_{1}$ and $M_{n}^{\prime} \in\left[M_{n}\right]^{\mathrm{N}_{1}}$ such that

$$
\operatorname{tp}\left(K_{0}(\mu) \cap E_{n \rho_{n}}\right)<\omega_{1}^{\omega+1} \quad\left(\mu \in M_{n}{ }^{\prime}\right) .
$$

Therefore, by Lemma 2, for each $\mu \in M_{n}{ }^{\prime}$ there are $p_{n}(\mu)<\omega$ and $\sigma_{n}(\mu)<\omega_{1}$ such that

$$
\operatorname{tp}\left(K_{0}(\mu) \cap E_{n \rho_{n} \sigma}\right)<\omega_{1}^{p_{n}(\mu)} \text { for } \sigma_{n}(\mu)<\sigma<\omega_{1} .
$$

Now choose $M_{n+1} \in\left[M_{n}^{\prime}\right]^{\mathbb{N}_{1}}$ such that $p_{n}(\mu)=p_{n}\left(\mu \in M_{n+1}\right)$ and such that $A_{n}<M_{n+1}$.

Proceeding inductively in the above manner, we define sets $A_{n} \subset M_{0}$ and $E_{n} \subset C_{0}(n<\omega)$ such that (4.7) holds, $\operatorname{tp} A_{n}=\alpha_{n}, \operatorname{tp} E_{n \rho \sigma}=\omega_{1}^{\gamma_{n}}$,

Also, there are

$$
\begin{aligned}
& A_{0}<A_{1}<\ldots, \\
& E_{0}<E_{1}<\ldots, \\
& A_{m} \times E_{n} \subset K_{1} \quad(m \leqslant n<\omega) .
\end{aligned}
$$

$$
p_{n}<\omega, \rho_{n}<\omega_{1} \text { and } \sigma_{n}(\mu)<\omega_{1} \text { for } \mu \in A_{n}{ }^{\prime}=A_{n+1} \cup A_{n+2} \cup \ldots,
$$

such that

$$
\operatorname{tp}\left(K_{0}(\mu) \cap E_{n \rho_{n} \sigma}\right)<\omega_{1}^{p_{n}} \text { for } \sigma_{n}(\mu)<\sigma<\omega_{1} \text { and } \mu \in A_{n}{ }^{\prime} .
$$

Since $A_{n}{ }^{\prime}$ is countable, there is $\sigma_{n}<\omega_{1}$ such that $\sigma_{n}(\mu)<\sigma_{n}$ for all $\mu \in A_{n}{ }^{\prime}$. Put

$$
B_{n}=E_{n \rho_{n} \sigma_{n}}-\bigcup_{\mu \in A_{n^{\prime}}} K_{0}(\mu) .
$$

Since tp $\left(E_{n \rho_{n} \sigma_{n}}\right)=\omega_{1}^{\gamma_{n}}>\omega_{1}{ }^{\omega}$, it follows from (4.9) and (2.2) that tp $B_{n}=\omega_{1}{ }^{\gamma_{n}}$. Therefore, $B=B_{0} \cup B_{1} \cup \ldots(<)$ has type $\omega_{1}{ }^{\gamma}$. From (4.8) and (4.10) it follows that $A \times B \subset K_{1}$, where $A=A_{0} \cup A_{1} \cup \ldots(<)$. This completes the proof of the theorem, since tp $A=\alpha_{0}+\alpha_{1}+\ldots=\alpha^{\prime}$.

As we have already noted, the negative relation (4.4) shows that the condition placed on the cofinality type of $\gamma$ in Theorem 2 is essential. The next theorem shows that we can drop this condition if we strengthen the restriction on $\xi$ to $\xi \leqslant \omega_{1}{ }^{\omega+1}$. Theorem 3 shows that (4.4) is a best possible relation.

THeOREM 3. If $\alpha<\omega_{1}$ and $\omega+1 \leqslant \gamma<\omega_{2}$, then

$$
\left\{\begin{array}{c}
\omega_{1} \\
\omega_{1}^{\gamma}
\end{array}\right\} \rightarrow\left\{\begin{array}{cc}
1 & \alpha \\
\omega_{1}^{\omega+1} & \omega_{1}^{\gamma}
\end{array}\right\}
$$

Proof. In view of Theorem 2 we may assume that $\operatorname{co}\left(\omega_{1}{ }^{\gamma}\right)=\omega_{1}$. Let tp $C=\omega_{1}{ }^{\gamma}$ and let $K_{0} \cup K_{1}$ be any partition of $W_{1} \times C$ such that $\operatorname{tp}\left(K_{0}(\mu)\right)<\omega_{1}^{\omega+1}$ 
$\left(\mu<\omega_{1}\right)$. Since $\operatorname{co}\left(\omega_{1}{ }^{\gamma}\right)=\omega_{1}$, we may write

$$
C=\bigcup_{\rho<\omega_{1}} C_{\rho}(<),
$$

where tp $C_{\rho}=\omega_{1}{ }^{\gamma_{\rho}}\left(\rho<\omega_{1}\right)$ and $\omega \leqslant \gamma_{0} \leqslant \gamma_{1} \leqslant \ldots \leqslant \hat{\gamma}_{\omega_{1}}<\gamma$. By Lemma 2, there are $\rho(\mu)<\omega_{1}$ and $n(\mu)<\omega$ for $\mu<\omega_{1}$ such that

$$
\operatorname{tp}\left(F_{\mu} \cap C_{\rho}\right)<\omega_{1}^{n(\mu)} \quad\left(\rho(\mu)<\rho<\omega_{1}\right) .
$$

There is $M \in\left[W_{1}\right]^{\aleph_{1}}$ such that $n(\mu)=n(\mu \in M)$. Let $A$ be any subset of $M$ of order type $\alpha$ and chose $\rho_{0}<\omega_{1}$ such that $\rho(\mu)<\rho_{0}$ for all $\mu \in A$. By (2.2) and (2.3), we have

$$
\operatorname{tp}\left(C_{\rho}-\bigcup_{\mu \in A} K_{0}(\mu)\right)=\omega_{1}^{\gamma_{\rho}} \quad\left(\rho_{0}<\rho<\omega_{1}\right)
$$

and (4.11) follows.

It is easy to prove that, for $1 \leqslant \gamma \leqslant \omega+1$ and $\alpha<\omega_{1}$,

$$
\left\{\begin{array}{c}
\omega_{1} \\
\omega_{1}^{\gamma}
\end{array}\right\} \rightarrow\left\{\begin{array}{cc}
1 & \alpha \\
\omega_{1}^{\gamma} & \omega_{1}^{\gamma}
\end{array}\right\} .
$$

In Theorem 4 we establish a stronger result.

THEOREM 4. If $k<\omega, \alpha<\omega_{1}$ and $1 \leqslant \gamma \leqslant \omega+1$, then

$$
\left\{\begin{array}{c}
\omega_{1} \\
\omega_{1}{ }^{\gamma}
\end{array}\right\} \rightarrow\left\{\begin{array}{cc}
k & \alpha \\
\omega_{1}{ }^{\gamma} & \omega_{1}{ }^{\gamma}
\end{array}\right\} \text {. }
$$

Proof. We shall first prove (4.12) for the case $k=1$. If $\gamma=\omega+1$, then this is a special case of (4.11). Suppose $\gamma \leqslant \omega$. Let tp $C=\omega_{1}{ }^{\gamma}$ and let $W_{1} \times C=$ $K_{0} \cup K_{1}$ be a partition such that $\operatorname{tp}\left(K_{0}(\mu)\right)<\omega_{1}^{\gamma}\left(\mu<\omega_{1}\right)$. Since $\gamma \leqslant \omega$, it follows that there are $n<\omega$ and $M \in\left[W_{1}\right]^{{ }_{1}}$ such that $\operatorname{tp}\left(K_{0}(\mu)\right)<\omega_{1}{ }^{n}$ $(\mu \in M)$. Let $A$ be any subset of $M$ of order type $\alpha$. It follows from (2.2) and (2.3) that

$$
\operatorname{tp}\left(C-\bigcup_{\mu \in A} K_{0}(\mu)\right)=\omega_{1}^{\gamma}
$$

and so (4.12) holds with $k=1$.

Now assume $k>1$ and use induction. Let $W_{1} \times C=K_{0} \cup K_{1}$, where $\operatorname{tp} C=\omega_{1}{ }^{\gamma}$. Suppose that

$$
\operatorname{tp}\left(K_{0}(X)\right)<\omega_{1}^{\gamma}
$$

holds for any $X \in\left[W_{1}\right]^{k}$. If tp $\left(K_{0}(\mu)\right)<\omega_{1}{ }^{\gamma}$ for all $\mu \in W_{1}$, then, since (4.12) holds with $k=1$, there are $A \subset W_{1}$ and $B \subset C$ such that tp $A=\alpha$, 
tp $B=\omega_{1}{ }^{\gamma}$ and $A \times B \subset K_{1}$. Therefore, we may assume that $\operatorname{tp}\left(K_{0}\left(\mu_{0}\right)\right)=$ $\omega_{1}^{\gamma}$ for some $\mu_{0}<\omega_{1}$. Also, we have

$$
\operatorname{tp}\left(K_{0}\left(\mu_{0}\right) \cap K_{0}(X)\right)<\omega_{1}^{\gamma}
$$

for $X \in\left[W_{1}-\left\{\mu_{0}\right\}\right]^{k-1}$. By the induction hypothesis (4.12) holds if $k$ is replaced by $k-1$ and hence there are $A \subset W_{1}-\left\{\mu_{0}\right\}$ and $B \subset K_{0}\left(\mu_{0}\right)$ such that tp $A=\alpha, \operatorname{tp} B=\omega_{1}{ }^{7}$ and $A \times B \subset K_{1}$. This proves (4.12).

The cardinal relation (3, Theorem 32$)$

$$
\left\{\begin{array}{c}
\omega_{1} \\
\omega_{1}
\end{array}\right\} \rightarrow\left\{\begin{array}{ll}
\omega_{0} & \omega_{0} \\
\omega_{1} & \omega_{1}
\end{array}\right\}
$$

shows that $k$ cannot be replaced by $\omega$ in (4.12) so the result is best possible. It is natural to ask whether Theorems 1,2 and 3 can be strengthened by replacing 1 by $k(<\omega)$ in (4.2), (4.5) and (4.11) respectively. We know this is not possible in the case of Theorem 3 since we will show (Theorem 7), with the help of the continuum hypothesis, that

$$
\left\{\begin{array}{c}
\omega_{1} \\
\omega_{1}^{\gamma}
\end{array}\right\} \rightarrow\left\{\begin{array}{cc}
2 & \omega \\
\omega_{1}{ }^{\omega} & \omega_{1}{ }^{\gamma}
\end{array}\right\} \quad \begin{gathered}
\text { if } \omega+1<\gamma<\omega_{2} \text { and } \\
\operatorname{co} \omega_{1}{ }^{\gamma}=\omega_{1} .
\end{gathered}
$$

However, we do not know if Theorem 2 is best possible in the sense just described. The first problem of this kind which we cannot settle is:

Problem 1. Is the relation

$$
\left\{\begin{array}{c}
\omega_{1} \\
\omega_{1}^{\omega 2}
\end{array}\right\} \rightarrow\left\{\begin{array}{cc}
2 & \omega \\
\omega_{1}{ }^{\omega} & \omega_{1}{ }^{\omega 2}
\end{array}\right\}
$$

true or false?

We conclude this section by proving one further relation of this kind.

THEOREM 5. If $\alpha<\omega_{1}$ and $\beta<\omega_{1}{ }^{\omega+2}$, then

$$
\left\{\begin{array}{c}
\omega_{1} \\
\omega_{1}^{\omega+2}
\end{array}\right\} \rightarrow\left\{\begin{array}{cc}
2 & \alpha \\
\omega_{1}^{\omega+1} & \beta
\end{array}\right\} \text {. }
$$

Proof. Let tp $C=\omega_{1}{ }^{\omega+2}$ and let $W_{1} \times C=K_{0} \cup K_{1}$ be any partition. There is $\eta<\omega_{1}$ such that $\beta<\omega_{1}{ }^{\omega+1} \eta$. If $\operatorname{tp}\left(K_{0}(\mu)\right)<\omega_{1}{ }^{\omega+1} \eta$ for all $\mu \in W_{1}$, then, by Theorem 1, there are $A \subset W_{1}$ and $B \subset C$ such that $\operatorname{tp} A=\alpha, \operatorname{tp} B=\beta$ and $A \times B \subset K_{1}$. Therefore, we may assume that there is some $\mu_{0}<\omega_{1}$ such that $\operatorname{tp}\left(K\left(\mu_{0}\right)\right)=\omega_{1}{ }^{\omega+1} \eta$. Since $\operatorname{tp}\left(K\left(\mu_{0}\right) \cap K(\mu)\right)<\omega_{1}{ }^{\omega+1}\left(\mu \in W_{1}-\left\{\mu_{0}\right\}\right)$, it follows from Lemma 3 that there are $A \subset W_{1}-\left\{\mu_{0}\right\}$ and $B \subset K\left(\mu_{0}\right)$ such that $\operatorname{tp} A=\alpha, \operatorname{tp} B=\omega_{1}{ }^{\omega+1} \eta$ and $A \times B \subset K_{1}$. This proves the result.

We do not know if 2 can be replaced by 3 in (4.14). 
ProBlem 2. Does the relation

hold for $\alpha<\omega_{1}$ and $\beta<\omega_{1}{ }^{\omega+2}$ ?

$$
\left\{\begin{array}{c}
\omega_{1} \\
\omega_{1}{ }^{\omega+2}
\end{array}\right\} \rightarrow\left\{\begin{array}{cc}
3 & \alpha \\
\omega_{1}{ }^{\omega+1} & \beta
\end{array}\right\}
$$

\section{Lemmas}

We need the following three lemmas in order to prove Theorem 7 in the next section. Lemma 5 is essentially the same as Lemma 10.5 of (1) and Lemma 6 is a known result due to Erdös, Kakutani and Tukey (4). We repeat the short proofs of these for the convenience of the reader.

LEMMA 5. Let $0<|I| \leqslant \aleph_{0},\left|M_{i}\right|=\aleph_{0}(i \in I)$ and let

$$
M=\bigcup_{i \in I} M_{i}=\left\{\mu_{0}, \mu_{1}, \ldots, \hat{\mu}_{\omega}\right\}_{\neq} .
$$

If tp $S<\omega_{2}$, then there are sets $A_{\mu} \subset S(\mu \in M)$ such that

$$
\begin{aligned}
& \text { (i) } \operatorname{tp} A_{\mu_{n}}<\omega_{1}{ }^{n} \quad(n<\omega) \text {, } \\
& \text { (ii) }\left|\left\{\mu \in M: x \in A_{\mu}\right\}\right|<\aleph_{0} \text { for } x \in S \text {, } \\
& \text { (iii) } \operatorname{tp}\left(S-\bigcup_{\mu \in M_{i}} A_{\mu}\right)<\omega_{1}{ }^{\omega} \quad(i \in I) .
\end{aligned}
$$

Proof. Since the sets $M_{i}(i \in I)$ are infinite, there are mutually disjoint infinite sets $M_{i}^{\prime} \subset M_{i}(i \in I)$. Let $M_{i}^{\prime}=\left\{\mu_{n_{i j}}: j<\omega\right\}_{\neq}$, where $n_{i 0}<n_{i 1}<\ldots(i \in I)$.

By (2.4) there is a partition of $S$ into disjoint sets $C_{n}(n<\omega)$ such that $\operatorname{tp} C_{n}<\omega_{1}^{n}(n<\omega)$. Let $r<\omega$. If $r=n_{i j}$ for some pair $i, j$ with $i \in I$ and $0<j<\omega$, then we put

$$
A_{\mu_{r}}=\bigcup_{n_{i, j-1} \leqslant n \leqslant n_{i, j}} C_{n} \text {. }
$$

Otherwise, if

$$
\mu_{r} \notin \bigcup_{i \in I}\left(M_{i}^{\prime}-\left\{\mu_{i 0}\right\}\right),
$$

then we put $A_{\mu_{r}}=\varnothing$. In either case, tp $A_{\mu_{r}}<\omega_{1}^{r}(r<\omega)$ by (2.2), and (i) holds. If $x \in S$, then there is a unique $n<\omega$ such that $x \in C_{n}$. But there are only finitely many pairs $i, j$ with $i \in I$ and $0<j<\omega$ such that $n_{i j-1} \leqslant n \leqslant n_{i j}$ and so (ii) holds. Finally, (iii) holds since

$$
S-\bigcup_{\mu \in M_{i}} A_{\mu} \subset \bigcup_{n<n_{i 0}} C_{n}=D
$$

and the order type of $D$ is less than $\omega_{1}^{n_{i 0}}$ by (2.2). 
LEMMA 6. The complete graph on $W_{1}$ is the union of $\aleph_{0}$ forests, i.e. there is a partition $\left[W_{1}\right]^{2}=E_{0} \cup E_{1} \cup \ldots \cup \hat{E}_{\omega}$ such that the graph $T_{i}=\left(W_{1}, E_{i}\right)(i<\omega)$ contains no circuit.

Proof. For $\mu<\omega_{1}$ there is $t=t(\mu) \leqslant \omega$ such that $\{v: v<\mu\}=\left\{v_{\mu 0}, v_{\mu 1}, \ldots\right.$, $\left.\hat{v}_{\mu t}\right\}_{\neq}$. Thus, for $v<\mu$, there is an unique $i<t(\mu)$ such that $v=v_{u i}$. Put

$$
E_{i}=\bigcup_{\mu<\omega_{1}}\left\{v_{\mu i}, \mu\right\}_{<}
$$

Then

$$
\left[W_{1}\right]^{2}=\bigcup_{i<\omega} E_{i}
$$

Suppose that $T_{i}=\left(W_{i}, E_{i}\right)$ contains a circuit. Then there are $\left\{v, v^{\prime}, \mu\right\}_{<} \subset W_{1}$ such that $\{v, \mu\}_{<} \in E_{i}$ and $\left\{v^{\prime}, \mu\right\}_{<} \in E_{i}$. But this implies the contradiction $v=v^{\prime}=v_{\mu i}$.

LEMMA 7. Let $\left|N_{i}\right|=\aleph_{0}(i<\omega)$ and let $G_{i}=\left(S, E_{i}\right)(i<\omega)$ be a graph without circuits of length 4 . Then there are disjoint sets $K_{i}(i<\omega)$ such that

$$
\begin{aligned}
& \text { (i) } K_{i} \in\left[N_{0} \cup N_{1} \cup \ldots \cup N_{i}\right]^{\leqslant i+1} \text {, } \\
& \text { (ii) } K_{i} \cap N_{j} \neq \varnothing \quad(j \leqslant i), \\
& \text { (iii) }\left[K_{i}\right]^{2} \cap E_{j}=\varnothing \quad(j \leqslant i) .
\end{aligned}
$$

Proof. We will say that a set $K$ has property $P_{n i}(i \leqslant n<\omega)$ if the conditions (i) and (ii) of the lemma are satisfied with $K_{i}=K$ and if

$$
\text { (iii) }^{\prime}[K]^{2} \cap E_{j}=\varnothing \quad(j \leqslant n) .
$$

This last condition is stronger than (iii) since $i \leqslant n$. We will prove that for fixed $i$ and $n(i \leqslant n<\omega)$, there are infinitely many mutually disjoint sets having property $P_{n i}$.

If $i=0$, this is obvious since each one-element subset of $N_{0}$ has property $P_{n 0}$. Now assume that $0<i \leqslant n<\omega$ and use induction on $i$. By assumption there are infinitely many mutually disjoint sets with property $P_{n, i-1}$ and we choose any $n+2$ of these, say $L_{0}, L_{1}, \ldots, L_{n+1}$. We claim that, if $F \in\left[N_{i}\right]^{t}$ and $t>\frac{1}{2}(n+2)(n+1)^{2} i^{2}$, then there are $x \in F$ and $\rho \leqslant n+1$ such that $L_{\rho} \cup\{x\}$ has property $P_{n i}$. Suppose this is false. Then, for each $x \in F$ and $\rho \leqslant n+1$ there are $j(\rho, n) \leqslant n$ and $y(\rho, x) \in L_{\rho}$ such that

$$
\{y(\rho, x), x\}_{\neq} \in E_{j(\rho, x)} .
$$

This follows since (i) and (ii) hold for $L_{\rho} \cup\{x\}$ and (iii)' holds for $L_{\rho}$. There 
are $\rho_{1}(x), \rho_{2}(x) \leqslant n+1$ such that $\rho_{1}(x) \neq \rho_{2}(x)$ and $j\left(\rho_{1}(x), x\right)=$ $j\left(\rho_{2}(x), x\right)=j(x)$. There are at most $\frac{1}{2}(n+2)(n+1)^{2} i^{2}$ different vectors

$$
v(x)=\left(\rho_{1}(x), \rho_{2}(x), j(x), y\left(\rho_{1}(x), x\right), y\left(\rho_{2}(x), x\right)\right)
$$

and hence, there are $x_{1}, x_{2} \in F$ such that $x_{1} \neq x_{2}$ and $v\left(x_{1}\right)=v\left(x_{2}\right)=$ $\left(\rho_{1}, \rho_{2}, j, y_{1}, y_{2}\right)$. Note that $\rho_{1} \neq \rho_{2}$ and hence $y_{1} \neq y_{2}$ since $y_{1} \in L_{\rho_{1}}, y_{2} \in L_{\rho_{2}}$ and $L_{\rho_{1}}, L_{\rho_{2}}$ are disjoint. Therefore, $\left\{x_{1}, y_{1}, x_{2}, y_{2}\right\}_{\neq}$is a circuit in $G_{j}$ of length 4 . This contradiction proves our claim, i.e. there are $x \in F$ and $\rho \leqslant n+1$ such that $J_{0}=L_{\rho \cup}\{x\}$ has property $P_{n i}$. This argument may be repeated choosing another set $L_{0}{ }^{\prime}, \ldots, L_{n+1}^{\prime}$ of $n+2$ sets with property $P_{n, i-1}$ so that these are mutually disjoint and disjoint from $J_{0}$. As before, there are $x^{\prime} \in N_{i}-J_{0}$ and $p^{\prime} \leqslant n+1$ such that $J_{1}=L_{\rho^{\prime} \cup}^{\prime}\left\{x^{\prime}\right\}$ has property $P_{n i}$. In this way we construct infinitely many mutually disjoint sets with property $P_{n i}$. The assertion of the previous paragraph now follows by induction.

In particular, there are infinitely many mutually disjoint sets having property $P_{i i}(i<\omega)$. Therefore, we can choose the finite sets $K_{i}(i<\omega)$ so that (i), (ii) and (iii) hold and so that these are mutually disjoint.

\section{Negative relations}

In Theorem 6 we establish the negative polarized partition relation (4.4) discussed in $\S 4$. The condition placed upon the cofinality type of $\omega_{1}^{\gamma}$ is necessary by Theorem 2 .

THEOREM 6. If $\gamma<\omega_{2}$ and $\operatorname{co}\left(\omega_{1}{ }^{\gamma}\right)=\omega_{1}$, then

$$
\left\{\begin{array}{l}
\omega_{1} \\
\omega_{1}^{\gamma}
\end{array}\right\} \rightarrow\left\{\begin{array}{cc}
1 & \omega \\
\omega_{1}{ }^{\omega+1}+1 & \omega_{1}^{\gamma}
\end{array}\right\} .
$$

Proof. Let $S$ be an ordered set of type $\omega_{1}{ }^{\gamma}$. Then we may write

$$
S=S_{0} \cup S_{1} \cup \ldots \cup \hat{S}_{\omega_{1}}(<),
$$

where $\operatorname{tp} S_{v}=\omega_{1}{ }^{\gamma_{v}}$ and $\gamma_{0} \leqslant \gamma_{1} \leqslant \ldots \leqslant \hat{\gamma}_{\omega_{1}} \leqslant \gamma$. By (2.4) there is a partition of $S_{v}$,

$$
S_{v}=\bigcup_{v<\omega} A_{v n},
$$

with $\operatorname{tp} A_{v n}<\omega_{1}{ }^{n}\left(v<\omega_{1} ; n<\omega\right)$. For $v<\omega_{1}$, there is a mapping $f_{v}$ of $\{n: n<\omega\}$ onto $\{\mu: \mu \leqslant v\}$, and for each $\mu \leqslant v$ there is an integer $n=n(\mu, v)$ such that $\mu=f_{v}(n)$. Now consider the partition $W_{1} \times S=K_{0} \cup K_{1}$ in which

$$
K_{0}(\mu)=\bigcup_{\mu \leqslant v<\omega_{1}} \bigcup_{n<n(\mu, v)} A_{v n}
$$

for $\mu<\omega_{1}$. 
By (2.2), $\operatorname{tp}\left(K_{0}(\mu) \cap S_{v}\right)<\omega_{1}{ }^{n(\mu, v)}<\omega_{1}{ }^{\omega}$ if $\mu \leqslant v<\omega_{1}$. Therefore, since $K_{0}(\mu) \cap S_{v}=\varnothing\left(v<\mu<\omega_{1}\right)$, we have

$$
\operatorname{tp} K_{0}(\mu) \leqslant \omega_{1}{ }^{\omega+1} .
$$

Let $N \in\left[W_{1}\right]^{N o}$. Then $N$ contains an increasing sequence of ordinals $\mu_{i}(i<\omega)$. Let

$$
\lambda=\lim _{i<\omega} \mu_{i} \text {. }
$$

For $v \geqslant \lambda$, the integers $n\left(\mu_{i}, v\right)(i<\omega)$ are all distinct and therefore, by (6.2),

$$
S_{v} \subset \bigcup_{i<\omega} K_{0}\left(\mu_{i}\right) \quad\left(\lambda \leqslant v<\omega_{1}\right)
$$

Therefore

$$
\bigcap_{\mu \in N} K_{1}(\mu) \subset S_{0} \cup S_{1} \cup \ldots \cup \hat{S}_{\lambda},
$$

and

$$
\operatorname{tp}\left\{\bigcap_{\mu \in N} K_{1}(\mu)\right\}<\omega_{1}^{\gamma} .
$$

The theorem follows from (6.3) and (6.4).

We now establish a much stronger result than ( 6.1$)$ by using the continuum hypothesis. Note that (6.5) implies (4.13).

$\left(^{*}\right)$ THEOREM 7. If $\omega+1<\gamma<\omega_{2}$ and $\operatorname{co}\left(\omega_{1}{ }^{\gamma}\right)=\omega_{1}$, then

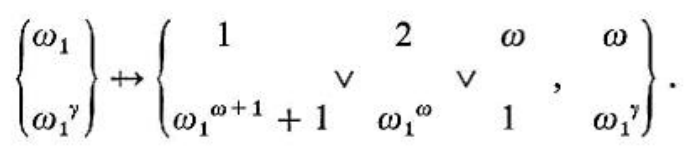

Proof. Let tp $S=\omega_{1}{ }^{\gamma}$. In order to prove (6.5) it is enough to construct sets $F_{\mu} \subset S\left(\mu<\omega_{1}\right)$ such that

(i) $\operatorname{tp} F_{\mu} \leqslant \omega_{1}{ }^{\omega+1} \quad\left(\mu<\omega_{1}\right)$,

(ii) $\operatorname{tp}\left(F_{\mu} \cap F_{\lambda}\right)<\omega_{1}^{\omega} \quad\left(\mu<\lambda<\omega_{1}\right)$,

(iii) $\left\{\mu<\omega_{1}: x \in F_{\mu}\right\}$ is finite for each $x \in S$, and

(iv) tp $\left(S-\bigcup_{\mu \in N} F_{\mu}\right)<\omega_{1}{ }^{\gamma}$ whenever $N \in\left[W_{1}\right]^{N_{0}}$.

Let $S=S_{0} \cup S_{1} \cup \ldots \cup \hat{S}_{\omega_{1}}(<)$, where tp $S_{v}=\omega_{1}^{\gamma_{v}}<\omega_{1}^{\gamma}$. By Lemma 5 , for each $v<\omega_{1}$ there are sets $A_{v n} \subset S_{v}(n<\omega)$ such that 


$$
\begin{gathered}
\operatorname{tp} A_{v n}<\omega_{1}{ }^{n} \quad(n<\omega), \\
\left\{n<\omega: x \in A_{v n}\right\} \text { is finite for } x \in S_{v}, \\
\operatorname{tp}\left(S_{v}-\bigcup_{n_{0}<n<\omega} A_{v n}\right)<\omega_{1}{ }^{\omega} .
\end{gathered}
$$

By the continuum hypothesis, $\left[W_{1}\right]^{\aleph_{0}}=\left\{M_{0}, M_{1}, \ldots, M_{\omega_{1}}\right\}_{\neq}$. For $v<\omega_{1}$, let $\left\{M_{0}, M_{1}, \ldots, M_{v}\right\}_{\neq}=\left\{N_{v 0}, N_{v 1}, \ldots, \hat{N}_{v \omega}\right\}$ (the sets $N_{v n}(n<\omega)$ are not necessarily different). By Lemma 6 , there is a partition of $\left[W_{1}\right]^{2}$ into $\aleph_{0}$ sets,

$$
\left[W_{1}\right]^{2}=E_{0} \cup E_{1} \cup \ldots \cup \hat{E}_{\omega},
$$

such that each graph $T_{i}=\left(W_{1}, E_{i}\right)(i<\omega)$ is a forest. By Lemma 7 , there are disjoint sets $K_{v n}(n<\omega)$ such that

$$
\begin{gathered}
K_{v n} \subset\left[N_{v 0} \cup \ldots \cup N_{v n}\right]^{\leqslant n+1}, \\
K_{v n} \cap N_{v j} \neq \varnothing \quad(j \leqslant n), \\
{\left[K_{v n}\right]^{2} \cap E_{j}=\varnothing \quad(j \leqslant n) .}
\end{gathered}
$$

We shall define the sets $F_{\mu}\left(\mu<\omega_{1}\right)$ by describing the intersections $F_{\mu} \cap S_{v}$ $\left(\mu, v<\omega_{1}\right)$. Let $\mu, v<\omega_{1}$ be fixed. Since the sets $K_{v n}(n<\omega)$ are mutually disjoint, there is at most one integer $n$ such that $\mu \in K_{v n}$. If $\mu \in K_{v n}$, then we define $F_{\mu} \cap S_{v}=A_{v n}$. If, on the other hand,

$$
\mu \notin \bigcup_{n<\infty} K_{v n},
$$

then we put $F_{\mu} \cap S_{v}=\varnothing$. This defines the sets $F_{\mu}\left(\mu<\omega_{1}\right)$ and we have to verify that (i)-(iv) hold.

Clearly (i) holds since $\operatorname{tp}\left(F_{\mu} \cap S_{v}\right)<\omega_{1}{ }^{\omega}\left(\mu, v<\omega_{1}\right)$. Let $\mu<\lambda<\omega_{1}$. There is a unique integer $l$ such that $\{\mu, \lambda\}<$ is an edge of $T_{l}$, i.e. such that $\{\mu, \lambda\} \in E_{l}$. Therefore, by (6.11), if $v<\omega_{1}$ and $l \leqslant n<\omega$, then $\mu$ and $\lambda$ are not both elements of $K_{v n}$. It follows from this and the way $F_{\mu} \cap S_{v}$ and $F_{\lambda} \cap S_{v}$ are defined, that

$$
F_{\mu} \cap F_{\lambda} \cap S_{v} \subset A_{v 0} \cup \ldots \cup \hat{A}_{v l}
$$

for all $v<\omega_{1}$. Therefore, $\operatorname{tp}\left(F_{\mu} \cap F_{\lambda} \cap S_{v}\right)<\omega_{1}^{l}$ by (6.6) and (2.2). Thus, $\operatorname{tp}\left(F_{\mu} \cap F_{\lambda}\right) \leqslant \omega_{1}{ }^{l+1}<\omega_{1}{ }^{\omega}$ and (ii) holds.

If $x \in S$, then there is a unique $y<\omega_{1}$ such that $x \in S_{y}$. By (6.7), there is a finite set of integers $N(x)$ such that $x \notin A_{v n}$ if $n \notin N(x)$. From the definition of the sets $F_{\mu} \cap S_{v}$, it follows that $x \notin F_{\mu}$ unless

$$
\mu \in \bigcup_{n \in N(x)} K_{v n} .
$$

This proves that (iii) holds since the sets $K_{v n}$ are finite. 
Finally, let $N \in\left[W_{1}\right]^{N_{0}}$. Then $N=M_{\sigma}$ for some $\sigma<\omega_{1}$. Let $\sigma \leqslant v<\omega_{1}$. Then there is an integer $j(\sigma, v)$ such that $N=N_{v, j(\sigma, v)}$. By (6.10) there is an element

$$
\mu_{n} \in K_{v n} \cap N_{v, j(\sigma, v)}
$$

for $j(\sigma, v) \leqslant n<\omega$. Therefore, by the definition of $F_{\mu_{n}}$,

$$
A_{v n} \subset F_{\mu_{n}} \subset \bigcup_{\mu \in N} F_{\mu} \quad(j(\sigma, v) \leqslant n<\omega) .
$$

Therefore, by (6.8),

$$
\operatorname{tp}\left(S_{v}-\bigcup_{\mu \in N} F_{\mu}\right)<\omega_{1}^{\omega} \quad\left(\sigma \leqslant v<\omega_{1}\right) .
$$

It follows that

$$
\operatorname{tp}\left(S-\bigcup_{\mu \in N} F_{\mu}\right) \leqslant \sum_{v<\sigma} \omega_{1}^{{ }^{\gamma} \sigma}+\omega_{1}{ }^{\omega+1}<\omega_{1}{ }^{\gamma} .
$$

This proves (iv) and completes the proof of Theorem 7.

We proved in (1, Theorem 10.14) with the continuum hypothesis that

$$
\left\{\begin{array}{l}
\omega \\
\lambda
\end{array}\right\} \rightarrow\left\{\begin{array}{ccccc}
1 & & \omega & & \omega \\
& \vee & & , & \\
\omega_{1}^{\omega} & & 1 & & \omega_{1}^{\omega+2}
\end{array}\right\}
$$

holds for all $\lambda<\omega_{2}$. This result is best possible in the sense that none of the entries on the right side of (6.12) can be decreased (see §7). We shall use (6.12) to establish an analogous result for $\left(\omega_{1}, \lambda\right)$-systems.

(*) THEOREM 8. If $\lambda<\omega_{2}$, then

$$
\left\{\begin{array}{c}
\omega_{1} \\
\lambda
\end{array}\right\} \rightarrow\left\{\begin{array}{ccccc}
1 & & \omega & \omega \\
\omega_{1}^{\omega+2} & & 1 & \omega_{1}^{\omega+2}
\end{array}\right\} .
$$

Proof. It is enough to prove (6.13) for the case when $\lambda=\omega_{1}{ }^{\gamma}, \gamma<\omega_{2}$.

For $\gamma<\omega+2$, the result is immediately obvious. We shall, therefore, assume that $\gamma \geqslant \omega+2$ and use induction on $\gamma$.

Let tp $S=\omega_{1}{ }^{\gamma}$. We shall construct sets $F_{\mu} \subset S\left(\mu<\omega_{1}\right)$ such that

and

(i) $\operatorname{tp} F_{\mu}<\omega_{1}^{\omega+2} \quad\left(\mu<\omega_{1}\right)$,

(ii) $\left|\left\{\mu<\omega_{1}: x \in F_{\mu}\right\}\right|<\aleph_{0} \quad(x \in S)$,

(iii) $\operatorname{tp}\left(S-\bigcup_{\mu \in N} F_{\mu}\right)<\omega_{1}^{\omega+2}$ whenever $N \in\left[W_{1}\right]^{N_{0}}$. 
Case 1. $\operatorname{co}(\gamma)=\omega$. In this case $S=S_{0} \cup \ldots \hat{S}_{\omega}(<)$, where tp $S_{n}=\omega_{1}{ }^{\gamma_{n}}<\omega_{1}^{\gamma}$. By the induction hypothesis, there are sets $F_{n \mu} \subset S_{n}\left(\mu<\omega_{1} ; n<\omega\right)$ such that, for $n<\omega$,

$$
\begin{gathered}
\operatorname{tp} F_{n \mu}<\omega_{1}^{\omega+2} \quad\left(\mu<\omega_{1}\right), \\
\left|\left\{\mu<\omega_{1}: x \in F_{n \mu}\right\}\right|<\aleph_{0} \quad\left(x \in S_{n}\right), \\
\operatorname{tp}\left(S_{n}-\bigcup_{\mu \in N} F_{n \mu}\right)<\omega_{1}^{\omega+2} \text { for } N \in\left[W_{1}\right]^{\aleph_{0}} .
\end{gathered}
$$

It is easy to verify that the sets $F_{\mu}=\bigcup_{n<\omega} F_{n \mu}$ satisfy all the conditions (i), (ii) and (iii).

The next case is less trivial.

Case 2. $\operatorname{co}\left(\omega_{1}{ }^{\gamma}\right)=\omega_{1}$.

In this case,

$$
S=\bigcup_{\nu<\omega_{1}} S_{\nu}(<),
$$

where $\operatorname{tp} S_{v}=\omega_{1}^{\gamma_{v}}<\omega_{1}^{\gamma}\left(v<\omega_{1}\right)$. Therefore, by the induction hypothesis, there are sets $F_{v \mu}^{1} \subset S_{v}\left(\mu<\omega_{1} ; v<\omega_{1}\right)$ such that

$$
\begin{array}{cc}
\operatorname{tp} F_{v \mu}^{1}<\omega_{1}^{\omega+2} & \left(\mu, v<\omega_{1}\right), \\
\left|\left\{\mu<\omega_{1}: x \in F_{v \mu}^{1}\right\}\right|<\aleph_{0} & \left(x \in S_{v} ; v<\omega_{1}\right), \\
\operatorname{tp}\left(S_{v}-\bigcup_{\mu \in N} F_{v \mu}^{1}\right)<\omega_{1}^{\omega+2} & \left(N \in\left[W_{1}\right]^{\aleph_{0}} ; v<\omega_{1}\right) .
\end{array}
$$

By the continuum hypothesis, $\left[W_{1}\right]^{N_{0}}=\left\{N_{0}, N_{1}, \ldots, \widehat{N}_{\omega_{1}}\right\}_{\neq}$. From Lemma 5 it follows that for each $v<\omega_{1}$, there are $\aleph_{0} \operatorname{sets} F_{v \mu}^{2} \subset S_{v}\left(\mu \in N_{0} \cup \ldots \cup N_{v}\right)$ such that

$$
\begin{gathered}
\operatorname{tp} F_{v \mu}^{2}<\omega_{1}^{\omega} \quad\left(\mu \in N_{0} \cup \ldots \cup N_{v} ; v<\omega_{1}\right), \\
\left|\left\{\mu \in N_{0} \cup \ldots \cup N_{v}: x \in F_{v \mu}^{2}\right\}\right|<\aleph_{0} \quad\left(x \in S_{v} ; v<\omega_{1}\right), \\
\operatorname{tp}\left(S_{v}-\bigcup_{\mu \in N_{\rho}} F_{v \mu}^{2}\right)<\omega_{1}^{\omega} \quad\left(\rho \leqslant v<\omega_{1}\right) .
\end{gathered}
$$

By (6.12) $\dagger$, there are sets $F_{v \mu}^{3} \subset S_{v}\left(\mu \leqslant v<\omega_{1}\right)$ such that

$$
\begin{gathered}
\operatorname{tp} F_{v \mu}^{3}<\omega_{1}^{\omega} \quad\left(\mu \leqslant v<\omega_{1}\right), \\
\left|\left\{\mu \leqslant v: x \in F_{v \mu}^{3}\right\}\right|<\aleph_{0} \quad\left(x \in S_{v} ; v<\omega_{1}\right), \\
\operatorname{tp}\left(S_{v}-\bigcup_{\mu \in N} F_{v \mu}^{3}\right)<\omega_{1}^{\omega+2} \quad\left(N \in[\{0,1, \ldots, v\}]^{\aleph_{0}} ; v<\omega_{1}\right) .
\end{gathered}
$$

$\dagger$ Note that we can only properly apply (6.12) when $\omega \leqslant v<\omega_{1}$. However, for $v<\omega$, if we put $F^{3}{ }_{\nu \mu}=\varnothing(\mu \leqslant v)$, then (6.20), (6.21) and (6.22) are all satisfied, the last vacuously. 
Now define the sets $F_{\mu}\left(\mu<\omega_{1}\right)$ by putting

$$
F_{\mu}=\bigcup_{v<\mu} F_{v \mu}^{1} \cup\left(\bigcup_{\nu<\omega_{1} 1} \bigcup_{\mu \in N_{0} \cup \ldots \cup N_{v}} F_{v \mu}^{2}\right) \cup \bigcup_{\mu \leqslant v<\omega_{1}} F_{v \mu}^{3} .
$$

We shall verify that (i), (ii) and (iii) hold.

By (6.14) and (6.17) we have that

$$
\operatorname{tp}\left(F_{\mu} \cap S_{v}\right)<\omega_{1}^{\omega+2} \quad(v<\mu),
$$

and by (6.17) and (6.20)

$$
\operatorname{tp}\left(F_{\mu} \cap S_{v}\right)<\omega_{1}^{\omega} \quad(\mu \leqslant v) .
$$

This implies that $\operatorname{tp} F_{\mu}<\omega_{1}^{\omega+2}\left(\mu<\omega_{1}\right)$, i.e. (i) holds.

Let $x \in S$. Then there is a unique $v<\omega_{1}$ such that $x \in S_{v}$. By (6.15), (6.18) and (6.21) it follows that $x$ is a member of only finitely many of the sets $F_{v \mu}^{\rho}(\rho=1,2$ or 3$)$ and hence (ii) holds.

Let $N \in\left[W_{1}\right]^{N_{0}}$. Then there is $\rho<\omega_{1}$ such that $N_{\rho}=\left\{\mu_{0}, \mu_{1}, \ldots, \hat{\mu}_{\omega}\right\}_{<} \subset N$. Let $\lambda=\lim _{n<\infty} \mu_{n}$. If $v<\lambda$, then $N^{\prime}=\left\{\mu_{n}: n<\omega ; v<\mu_{n}\right\}$ is infinite and therefore, by (6.16),

$$
\operatorname{tp}\left(S_{v}-\bigcup_{\mu \in N} F_{\mu}\right) \leqslant \operatorname{tp}\left(S_{v}-\bigcup_{\mu \in N^{\prime}} F_{v \mu}^{1}\right)<\omega_{1}^{\omega+2} .
$$

If $\rho \leqslant v<\omega_{1}$, then by (6.19),

$$
\operatorname{tp}\left(S_{v}-\bigcup_{\mu \in N} F_{\mu}\right) \leqslant \operatorname{tp}\left(S_{v}-\bigcup_{\mu \in N_{\rho}} F_{v \mu}^{2}\right)<\omega_{1}^{\omega} .
$$

Also, if $\lambda \leqslant v<\rho$, then $N_{\rho} \in[\{0,1,2, \ldots, v\}]^{\text {No }}$ and

$$
\operatorname{tp}\left(S_{v}-\bigcup_{\mu \in N} F_{\mu}\right) \leqslant \operatorname{tp}\left(S_{v}-\bigcup_{\mu \in N_{\rho}} F_{v \mu}^{3}\right)<\omega_{1}^{\omega+2}
$$

by (6.22). Let $\pi=\max \{\lambda, \rho\}$. Then (6.23), (6.24) and (6.25) show that

$$
\operatorname{tp}\left(S_{v}-\bigcup_{\mu \in N} F_{\mu}\right)<\omega_{1}^{\omega+2} \quad(\mu<\pi)
$$

and

$$
\operatorname{tp}\left(S_{v}-\bigcup_{\mu \in N} F_{\mu}\right)<\omega_{1}^{\omega} . \quad\left(\pi \leqslant v<\omega_{1}\right) .
$$

Thus (iii) holds.

Theorem 8 now follows by induction on $\gamma$. 


\section{Conclusion}

We conclude by showing that $\omega_{1}^{\omega+2}$ in (6.13) cannot be replaced by anything smaller, i.e.

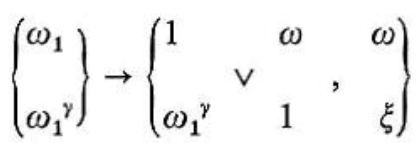

holds if $\xi<\omega_{1}^{\omega+2} \leqslant \omega_{1}^{\gamma}<\omega_{2}$. We remark that the situation is rather different with regard to the relation (6.12). For we have proved (1, Theorem 10.13) that

$$
\left\{\begin{array}{l}
\omega \\
\omega_{1}{ }^{\gamma}
\end{array}\right\} \rightarrow\left\{\begin{array}{llll}
1 & & \omega & \omega \\
& \vee & & , \\
\omega_{1}{ }^{\gamma} & 1 & \xi
\end{array}\right\}
$$

holds for $\xi<\omega_{1}^{\omega+2} \leqslant \omega_{1}^{\gamma}<\omega_{2}$ provided that

$$
\operatorname{co}(\gamma) \neq \omega \text { and } \operatorname{co}(\gamma-1) \neq \omega .
$$

Here $\gamma-1=\gamma^{\prime}$ if $\gamma=\gamma^{\prime}+1$ and $\gamma-1=\gamma$ if $\gamma$ is a limit ordinal. If (7.3) holds, then (7.2) is stronger than (7.1). However, if (7.3) is false, then (7.2) is also false (see Theorems 10.11 and 10.12 of (1)).

Proof of (7.1). In view of the remarks above, we can assume that (7.3) is false, i.e. either $\operatorname{co}(\gamma)=\omega$ or $\operatorname{co}(\gamma-1)=\omega$.

Let $\operatorname{tp} S=\omega_{1}{ }^{\gamma}$ and let $W_{1} \times S=K_{0} \cup K_{1}$ be a partition such that (i) $\operatorname{tp}\left(K_{0}(\mu)\right)<\omega_{1}^{\gamma}\left(\mu<\omega_{1}\right)$ and (ii) $\operatorname{tp}\left(K_{0}(x)\right)<\omega$ for $x \in S$. We have to show that there are sets $A \subset W_{1}$ and $B \subset S$ such that $\operatorname{tp} A \geqslant \omega, \operatorname{tp} B \geqslant \xi$ and $A \times B \subset K_{1}$.

Case 1. $\operatorname{co}(\gamma)=\omega$. In this case we may write $S=S_{0} \cup S_{1} \cup \ldots \cup \hat{S}_{\omega}(<)$, where tp $S_{n}=\omega_{1}^{\gamma_{n}+2}$ and $\omega \leqslant \gamma_{0}<\gamma_{1}<\ldots<\hat{\gamma}_{\omega}<\gamma$. For each $\mu<\omega_{1}$ there is an integer $n(\mu)$ such that

$$
\operatorname{tp}\left(K_{0}(\mu) \cap S_{n(\mu)}\right)<\omega_{1}^{\gamma_{n(\mu)}+2} .
$$

There is $M \subset W_{1}$ such that $\operatorname{tp} M=\omega$ and $n(\mu)=n(\mu \in M)$. Since (7.2) holds with $\gamma$ replaced by $\gamma_{n}+2$, it follows that there are $A \subset M$ and $B \subset S_{n(\mu)}$ such that $\operatorname{tp} A=\omega, \operatorname{tp} B=\xi$ and $A \times B \subset K_{1}$.

Case 2. $\gamma=\gamma^{\prime}+1$ and $c o\left(\gamma^{\prime}\right)=\omega$. We may write $S=S_{0} \cup \ldots \cup \hat{S}_{\omega_{1}}(<)$, where $\operatorname{tp} S_{v}=\omega_{1}{ }^{\gamma}\left(v<\omega_{1}\right)$. Let $\gamma_{n}(n<\omega)$ be a sequence of ordinals such that

$$
\omega \leqslant \gamma_{0}<\gamma_{1}<\ldots<\hat{\gamma}_{\omega}<\gamma^{\prime}=\lim _{n<\omega} \gamma_{n} .
$$


For $\mu<\omega_{1}$ there are $n(\mu)<\omega$ and $v(\mu)<\omega_{1}$ such that

$$
\operatorname{tp}\left(F_{\mu} \cap S_{v}\right)<\omega_{1}^{\gamma_{n(\mu)}} \quad\left(v(\mu)<v<\omega_{1}\right) .
$$

There is $M \subset W_{1}$ such that tp $M=\omega$ and $n(\mu)=n(\mu \in M)$. Choose $v_{0}<\omega_{1}$ so that $v(\mu)<v_{0}(\mu \in M)$ and let $S^{\prime}$ be a subset of $S_{v}$ of order type $\omega_{1}^{\gamma_{n}+2}$. As in Case 1 , there are $A \subset M$ and $B \subset S^{\prime}$ such that tp $A=\omega, \operatorname{tp} B=\xi$ and $A \times B \subset K_{1}$.

\section{References}

1. ERdös, P., Hajnal, A. and Mitner, E. C. On the complete subgraphs of graphs defined by systems of sets. Acta Math. Acad. Sci. Hung. 17 (1966), 159-229.

2. ERdös, P., HaJnal, A. and Milner, E. C. Set mappings and polarized partition relations. To appear in the Proceedings of the Balatonfüred Combinatorial Conference (1969).

3. ERdös, P., Hajnal, A. and Rado, R. Partition relations for cardinal numbers. Acta Math. Acad. Sci. Hung. 16 (1965), 93-196.

4. Erdös, P. and KakUtani, S. On non-denumerable graphs. Bull. Amer. Math. Soc. 49 (1943), 457-461.

5. ERDös, P. and RADo, R. Combinatorial theorems on classifications of subsets of a given set. Proc. London Math. Soc. (3) 2 (1952), 417-439.

6. ERdös, P. and Rado, R. A partition calculus in set theory. Bull. Amer. Math. Soc. 62 (1956), 427-489.

7. ERDös, P. and SPECKER, E. On a theorem in the theory of relations and a solution of a problem of Knaster. Colloq. Math. 8 (1961), 19-21.

8. Hajnal, A. Proof of a conjecture of Ruziewicz. Fund. Math. 50 (1961), 123-128.

9. MILNER, E. C. and RADO, R. The pigeon-hole principle for ordinal numbers. Proc. London Math. Soc. (3), 15 (1965), 750-768. 